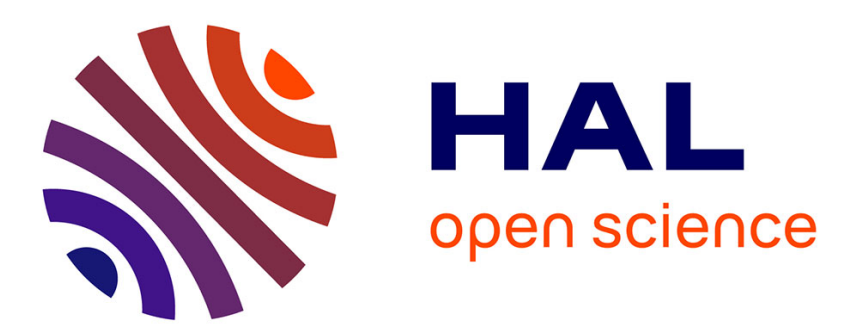

\title{
Electrochemical biosensors: recommended definitions and classification
}

Daniel R. Thevenot, Klara Toth, Richard Durst, George S. Wilson

\section{To cite this version:}

Daniel R. Thevenot, Klara Toth, Richard Durst, George S. Wilson. Electrochemical biosensors: recommended definitions and classification. Biosensors and Bioelectronics, 2001, 16, pp.121 - 131. 10.1081/AL-100103209 . hal-01084678

\section{HAL Id: hal-01084678 \\ https://hal-enpc.archives-ouvertes.fr/hal-01084678}

Submitted on 19 Nov 2014

HAL is a multi-disciplinary open access archive for the deposit and dissemination of scientific research documents, whether they are published or not. The documents may come from teaching and research institutions in France or abroad, or from public or private research centers.
L'archive ouverte pluridisciplinaire HAL, est destinée au dépôt et à la diffusion de documents scientifiques de niveau recherche, publiés ou non, émanant des établissements d'enseignement et de recherche français ou étrangers, des laboratoires publics ou privés.

\section{(c)(1)}

Distributed under a Creative Commons Attribution| 4.0 International License 


\title{
Technical report \\ Electrochemical biosensors: recommended definitions and classification $^{\text {t3 }}$
}

\author{
Daniel R. Thévenot ${ }^{\mathrm{a}, *, 1}$, Klara Toth ${ }^{\mathrm{b}}$, Richard A. Durst ${ }^{\mathrm{c}}$, George S. Wilson ${ }^{\mathrm{d}}$ \\ a Centre d'Enseignement et de Recherche sur l'Eau, la Ville et l'Environnement (Cereve), Faculté de Sciences et de Technologie, \\ Université Paris XII-Val de Marne, 61 Avenue du Général de Gaulle, 94010 Créteil Cedex, Paris, France \\ ${ }^{\mathrm{b}}$ Institute of General and Analytical Chemistry, Technical University of Budapest, Gellert ter 4, H1111, Budapest, Hungary \\ ${ }^{\mathrm{c}}$ Department of Food Science and Technology, Cornell University, Geneva, NY 14456-0462, USA \\ d Chemistry Department, Kansas University, Lawrence, KS 66045, USA
}

\begin{abstract}
Two Divisions of the International Union of Pure and Applied Chemistry (IUPAC), namely Physical Chemistry (Commission I.7 on Biophysical Chemistry formerly Steering Committee on Biophysical Chemistry) and Analytical Chemistry (Commission V.5 on Electroanalytical Chemistry) have prepared recommendations on the definition, classification and nomenclature related to electrochemical biosensors; these recommendations could, in the future, be extended to other types of biosensors. An electrochemical biosensor is a self-contained integrated device, which is capable of providing specific quantitative or semi-quantitative analytical information using a biological recognition element (biochemical receptor) which is retained in direct spatial contact with an electrochemical transduction element. Because of their ability to be repeatedly calibrated, we recommend that a biosensor should be clearly distinguished from a bioanalytical system, which requires additional processing steps, such as reagent addition. A device that is both disposable after one measurement, i.e. single use, and unable to monitor the analyte concentration continuously or after rapid and reproducible regeneration, should be designated a single use biosensor. Biosensors may be classified according to the biological specificity-conferring mechanism or, alternatively, to the mode of physico-chemical signal transduction. The biological recognition element may be based on a chemical reaction catalysed by, or on an equilibrium reaction with macromolecules that have been isolated, engineered or present in their original biological environment. In the latter cases, equilibrium is generally reached and there is no further, if any, net consumption of analyte(s) by the immobilized biocomplexing agent incorporated into the sensor. Biosensors may be further classified according to the analytes or reactions that they monitor: direct monitoring of analyte concentration or of reactions producing or consuming such analytes; alternatively, an indirect monitoring of inhibitor or activator of the biological recognition element (biochemical receptor) may be achieved. A rapid proliferation of biosensors and their diversity has led to a lack of rigour in defining their performance criteria. Although each biosensor can only truly be evaluated for a particular application, it is still useful to examine how standard protocols for performance criteria may be defined in accordance with standard IUPAC protocols or definitions. These criteria are recommended for authors, referees and educators and include calibration characteristics (sensitivity, operational and linear concentration range, detection and quantitative determination limits), selectivity, steady-state and transient response times, sample throughput, reproducibility, stability and lifetime. (C) 2001 Elsevier Science B.V. All rights reserved.
\end{abstract}

Keywords: Electrochemical biosensor; definition; single use biosensor; calibration characteristics; performance criteria; analytes

Abbreviations: Ab, antibody; Ag, antigen; BLM, bilayer lipid membrane; BSA, bovine serum albumin; CME, chemically modified electrode; ENFET, enzyme field-effect transistor; FET, field-effect transistor; FIA, flow injection analysis; HPLC, high performance liquid chromatography; IMFET, immunological field-effect transistor; ISE, ion selective electrode; ISFET, ion sensitive field-effect transistor; LP, lactose permease; $t L$, life time; LOD, limit of detection; LOQ, limit of quantification; NAD, nicotinamide adenine dinucleotide; PU, polyurethane; PVAL, poly(vinyl alcohol); SAM, self assembled monolayer; SPR, surface plasmon resonance; TCNQ; , tetracyanoquinodimethane; TTF + , tetrathiafulvalene.

Membership of the working party for the present project during the period 1993-1999 was as follows: D.R. Thévenot, R.P. Buck, K. Cammann, R.A. Durst, K. Toth and G.S. Wilson

* Corresponding author. Tel.: + 33-145-171625; fax: + 33-145-171627.

E-mail address: thevenot@univ-paris12.fr (D.R. Thévenot).

${ }^{1}$ International Union of Pure and Applied Chemistry: Physical Chemistry Division, Commission I.7 (Biophysical Chemistry); Analytical Chemistry Division, Commission V.5 (Electroanalytical Chemistry). 


\section{Definition and limitations}

\subsection{Biosensor}

A chemical sensor is a device that transforms chemical information, ranging from the concentration of a specific sample component to total composition analysis, into an analytically useful signal. Chemical sensors contain usually two basic components connected in series: a chemical (molecular) recognition system (receptor) and a physico-chemical transducer. Biosensors are chemical sensors in which the recognition system utilises a biochemical mechanism Cammann, 1977; Turner et al., 1987.

The biological recognition system translates information from the biochemical domain, usually an analyte concentration, into a chemical or physical output signal with a defined sensitivity. The main purpose of the recognition system is to provide the sensor with a high degree of selectivity for the analyte to be measured.
While all biosensors are more or less selective (non-specific) for a particular analyte, some are, by design and construction, only class-specific, since they use class enzymes, e.g. phenolic compound biosensors, or whole cells, e.g. used to measure biological oxygen demand. Because in sensing systems present in living organisms/ systems, such as olfaction, and taste, as well as neurotransmission pathways, the actual recognition is performed by cell receptor, the word receptor or bioreceptor is also often used for the recognition system of a chemical biosensor. Examples of single and multiple signal transfer are listed in Table 1. These examples are limited to the most common sensor principles, excluding existing laboratory instrumentation systems.

The transducer part of the sensor serves to transfer the signal from the output domain of the recognition system, mostly to the electrical domain. Because of the general significance of the word, a transducer provides bi-directional signal transfer (non-electrical to electrical and vice versa); the transducer part of a sensor is also

Table 1

Types of receptors used in biosensors and the electrochemical measurement techniques, linked to them that recognize specific species ${ }^{\mathrm{a}}$.

Analytes

\section{Ions}

2. Dissolved gases, vapours, odours

3. Substrates

4. Antibody/antigen

5. Various proteins and low molecular weight substrates, ions

Receptor/Chemical recognition system

\section{mixed valence metal oxides permselective, ion-conductive inorganic crystals trapped mobile synthetic or biological ionophores ion exchange glasses enzyme(s)}

bilayer lipid or hydrophobic membrane inert metal electrode enzyme(s) antibody, receptor

enzyme(s)

whole cells membrane receptors plant or animal tissue

antigen/antibody oligonucleotide duplex, aptamer enzyme labelled chemiluminescent or fluorescent labelled

specific ligands

protein receptors and channels enzyme labelled

fluorescent labelled
Measurement technique/ Transduction mode potentiometric, voltammetric

in series with 1 .

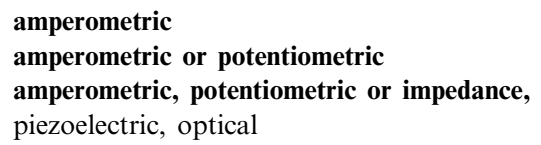
piezoelectric, optical, surface plasmon resonance in series with 3 . optical

as 4 .

\footnotetext{
a Biological receptors, which are part of electrochemical biosensors, are indicated in bold characters Bergveld and Thévenot, 1993. Besides quantification of the above mentioned analytes, biosensors are also used for detection and quantification of micro-organisms: receptors are bacteria, yeast or oligonucleotide probes coupled to electrochemical, piezoelectric, optical or calorimetric transducers.
} 
Table 2

Type of electrochemical transducers for classified type of measurements, with corresponding analytes to be measured Bergveld and Thévenot, 1993. ${ }^{\mathrm{a}}$

\begin{tabular}{lll}
\hline Measurement type & Transducer & Transducer analyte \\
\hline 1. Potentiometric & $\begin{array}{l}\text { ion-selective electrode (ISE) } \\
\text { glass electrode } \\
\text { gas electrode } \\
\text { metal electrode } \\
\text { metal or carbon electrode } \\
\text { chemically modified electrodes (CME) }\end{array}$ & $\begin{array}{l}\mathrm{K}^{+}, \mathrm{Cl}^{-}, \mathrm{Ca}^{2+}, \mathrm{F}^{-} \\
\mathrm{CO}_{2}^{+}, \mathrm{Na}^{+} \ldots \\
\mathrm{NH}_{3} \\
\text { redox species }\end{array}$ \\
$\begin{array}{lll}\text { 2. Amperometric } & \text { interdigitated electrodes, metal electrode } & \mathrm{O}_{2}, \text { sugars, alcohols... } \\
\text { sugars, alcohols, phenols, } \\
\text { oligonucleotides... }\end{array}$ \\
$\begin{array}{l}\text { 3. Conductometric, } \\
\text { impedimetric }\end{array}$ & $\begin{array}{l}\text { urea, charged species, oligonucleotides... } \\
\text { 4. Ion charge or field } \\
\text { effect }\end{array}$ & $\begin{array}{l}\mathrm{H}^{+}, \mathrm{K}^{+} \ldots \\
\end{array}$ \\
\hline
\end{tabular}

a Non electrochemical transducers are also used within biosensors: (a) piezoelectric (shear and surface acoustic wave); (b) calorimetric (thermistor); (c) optical (planar wave guide, fibre optic, surface plasmon resonance...)

called a detector, sensor or electrode, but the term transducer is preferred to avoid confusion. Examples of electrochemical transducers, which are often used for the listed types of measurement in Table 1, are given in Table 2, together with examples of analytes which have been measured. Transducers are classified by recognition element type (Table 1) or by electrochemical transducer mode (Table 2).

Finally, chemical sensors, as well as biosensors described below, are self-contained, all parts being packaged together in the same unit, usually small, the biological recognition element being in direct spatial contact with the transducing element.

\subsection{Electrochemical biosensor}

An electrochemical biosensor is a biosensor with an electrochemical transducer (Table 2). It is considered a chemically modified electrode (CME) Durst et al., 1997; Kutner et al., 1998 since electronic conducting, semiconducting or ionic conducting material is coated with a biochemical film.

A biosensor is an integrated receptor-transducer device, which is capable of providing selective quantitative or semi-quantitative analytical information using a biological recognition element. Thus, biological examples given in Table 1 are shown in bold characters.

A biosensor can be used to monitor either biological or non-biological matrixes. Chemical sensors, which incorporate a non-biological specificity-conferring part or receptor, although used for monitoring biological processes, as the in vivo $\mathrm{pH}$ or oxygen sensors, are not biosensors. These sensors are beyond the scope of the present report. Similarly, physical sensors used in biological environment, even when electrically based, such as in vivo pressure or blood flow sensors, are also excluded from this report.
Although biosensors with different transducer types, e.g. electrochemical, optical, piezoelectric or thermal types, show common features, this report is restricted to electrochemical biosensors (indicated in bold characters in Table 1). Optical, mass and thermal sensors will be described in future IUPAC reports. For example optical biosensors will be described by IUPAC commission V.4 in Spectrochemical and other optical procedures for analysis (project number $540 / 19$ / 95).

\subsection{Limitations in the use of the term 'biosensor'}

Since a biosensor is a self contained integrated device, we recommend that it should be clearly distinguished from an analytical system which incorporates additional separation steps such as high performance liquid chromatography (HPLC), or additional hardware and/or sample processing such as specific reagent introduction, as flow injection analysis (FIA). Thus, a biosensor should be a reagentless analytical device, although the presence of ambient co-substrates, such as water for hydrolases or oxygen for oxidoreductases, may be sufficient for the analyte determination. On the other hand, it may provide, as part of an integrated system, some separation or amplification steps achieved by inner or outer membranes or reacting layers. In conclusion, an HPLC or FIA system may incorporate a biosensor as a detecting device, and FIA is often convenient to evaluate the biosensor analytical performance (see Section 5.) On the contrary, an FIA system containing a reagent reservoir, an enzymatic or immunological reactor and, downstream, an electrochemical sensor, is not a biosensor.

Because of the importance of their ability to be repeatedly calibrated, we recommend that the term multiple-use biosensor be limited to devices suitable for monitoring both the increase and decrease of the ana- 
lyte concentrations in batch reactors or flow-through cells. Thus, single-use devices that cannot rapidly and reproducibly be regenerated should be named single-use biosensors. Various terms have been used for such disposable and non-regenerable devices, e.g. bioprobes, bioindicators. At present, none of these names have been generally accepted by the scientific community and we recommend designating them as single-use biosensors.

Finally, as is seen in the various sections of this report, the diversity of the molecular recognition systems and of the electrochemical transducers incorporated in each biosensor appears to be very wide. Nevertheless, common features, related to their operating principles, are significant. They mainly depend upon the type of transducer and molecular receptor used:

- because of the nature of their operational principle, amperometric sensors, including biocatalytic amperometric sensors, alter the concentration of the analyte in their vicinity; these sensors may reach a steady-state but they never reach equilibrium. Knowledge of the rate-limiting step of their response, i.e. mass transport rate versus analyte consumption reaction rate, is very important for understanding their operational characteristics;

- potentiometric as well as biocomplexing based sensors usually operate at or near equilibrium and are not subject to such transport limitations; on the other hand, the magnitude of their apparent equilibrium constant and kinetics, under experimental conditions, will define the continuity of the sensor response and the necessity for reagent introduction. If these sensors operate without requiring reagent addition and are capable of rapid and reproducible regeneration, then they are referred to as multipleuse biosensors.

\section{Classification}

Biosensors may be classified according to the biological specificity conferring mechanism, or to the mode of signal transduction or, alternatively, a combination of the two. These might also be described as amperometric, potentiometric, field-effect or conductivity sensors. Alternatively, they could be termed, for example, as amperometric enzyme sensors Inczedy et al., 1998. As an example, the former biosensors may be considered as enzyme- or immuno-sensors.

\subsection{Receptor: biological recognition element}

\subsubsection{Biocatalytic recognition element}

In this case, the biosensor is based on a reaction catalysed by macromolecules, which are present in their original biological environment, have been isolated pre- viously or have been manufactured. Thus, a continuous consumption of substrate(s) is achieved by the immobilized biocatalyst incorporated into the sensor: transient or steady-state responses are monitored by the integrated detector. Three types of biocatalyst are commonly used:

1. Enzyme (mono- or multi-enzyme), the most common and well developed recognition system,

2. Whole cells (micro-organisms, such as bacteria, fungi, eukaryotic cells or yeast) or cell organelles or particles (mitochondria, cell walls),

3. Tissue (plant or animal tissue slice).

The biocatalytic-based biosensors are the best known and studied and have been the most frequently applied to biological matrices since the pioneering work of Clark Clark et al., 1962. One or more analytes, usually named substrates $\mathrm{S}$ and $\mathrm{S}^{\prime}$, react in the presence of enzyme(s), whole cells or tissue culture and yield one or several products, $\mathrm{P}$ and $\mathrm{P}^{\prime}$, according to the general reaction scheme:

$\mathrm{S}+\mathrm{S}^{\prime} \stackrel{\text { biocatalyst }}{\longrightarrow} \mathrm{P}+\mathrm{P}^{\prime}$

There are four strategies that use adjacent transducers for monitoring the analyte $\mathrm{S}$ consumption by this biocatalysed reaction:

- detection of the co-substrate S' consumption, e.g., oxygen depleted by oxidase, bacteria or yeast reacting layers, and the corresponding signal decrease from its initial value;

- recycling of $\mathrm{P}$, one of the reaction products, e.g., hydrogen peroxide, $\mathrm{H}^{+}, \mathrm{CO}_{2}, \mathrm{NH}_{3}$, etc. production by oxidoreductase, hydrolase, lyase, etc., and corresponding signal increase;

- detection of the state of the biocatalyst redox active centre, cofactor, prosthetic group evolution in the presence of substrate $S$, using an immobilized mediator which reacts sufficiently rapidly with the biocatalyst and is easily detected by the transducer; various ferrocene derivatives as well as tetrathiafulvalene-tetracyanoquinodimethane $\left(\mathrm{TTF}^{+} \mathrm{TCNQ}^{-}\right.$) organic salt, quinones, quinoid dyes, $\mathrm{Ru}$ or Os complexes in a polymer matrix, have been used Bartlett et al., 1991;

- direct electron transfer between the active site of a redox enzyme and the electrochemical transducer.

The third strategy attempts to eliminate sensor response dependence on the co-substrate, $\mathrm{S}^{\prime}$, concentration and to decrease the influence of possible interfering species. The first goal is only reached when reaction rates are much higher for immobilized mediator with biocatalyst than those for co-substrate with biocatalyst. An alternative approach to the use of such mediators consists in restricting the analyte (substrate) concentration within the reaction layer through an appropriate outer membrane, whose permeability strongly favours 
co-substrate transport Scheller and Pfeiffer, 1978; Bindra et al., 1991.

When several enzymes are immobilized within the same reaction layer, several strategies for improving biosensor performance can be developed. Three following possibilities have been most frequently proposed:

- several enzymes facilitate the biological recognition by sequentially converting the product of a series of enzymatic reactions into a final electroactive form: this set-up allows a much wider range of possible biosensor analytes Wollenberger et al., 1993;

- multiple enzymes, applied in series, may regenerate the first enzyme co-substrate and a real amplification of the biosensor output signal may be achieved by efficient regeneration of another co-substrate of the first enzyme;

- multiple enzymes, applied in parallel, may improve the biosensor selectivity by decreasing the local concentration of electrochemical interfering substance: this set-up is an alternative to the use of either a permselective membrane (see Section 4.2) or a differential set-up, i.e., subtraction of the output signal generated by the biosensor and by a reference sensor having no biological recognition element Thévenot et al., 1979.

A recent development of enzyme based biosensors involves their operation in an organic solvent matrix: a hydrophilic microenvironment is often maintained within the enzyme and the substrate partitions between the matrix and the enzyme active site.

\subsubsection{Biocomplexing or bioaffinity recognition element}

The biosensor operation is based on interaction of the analyte with macromolecules or organized molecular assemblies that have either been isolated from their original biological environment or engineered Aizawa, 1991. Thus, equilibrium is usually reached and there is no further net consumption of the analyte by the immobilized biocomplexing agent. These equilibrium responses are monitored by the integrated detector. In some cases, this biocomplexing reaction is itself monitored using a complementary biocatalytic reaction. Steady-state or transient signals are then monitored by the integrated detector.

1. Antibody-antigen interaction. The most developed examples of biosensors using biocomplexing receptors are based on immunochemical reactions, i.e. binding of an antigen ( $\mathrm{Ag})$ to a specific antibody (Ab). Formation of such Ab-Ag complexes has to be detected under conditions where non-specific interactions are minimized. Each Ag determination requires the production of a particular $\mathrm{Ab}$, its isolation and, usually, its purification. Several studies have been described involving direct monitoring of the Ab-Ag complex formation on ion-sensitive-fieldeffect transistors (ISFETs). In order to increase the sensitivity of immuno-sensors, enzyme labels are frequently coupled to $\mathrm{Ab}$ or $\mathrm{Ag}$, thus requiring additional chemical synthesis steps. Even in the case of the enzyme-labelled $\mathrm{Ab}$, these biosensors will essentially operate at equilibrium, the enzymatic activity being there only to quantify the amount of complex produced. As the binding or affinity constant is usually very large, such systems are either irreversible (single-use biosensors) or placed within an FIA environment where $\mathrm{Ab}$ may be regenerated by dissociation of complexes by chaotropic agents, such as glycine- $\mathrm{HCl}$ buffer at $\mathrm{pH} 2.5$.

2. Receptor/antagonist/agonist. More recently, attempts have been made to use ion channels, membrane receptors or binding proteins as molecular recognition systems in conductometric, ISFET or optical sensors Sugawara et al., 1997a. For example, the transport, protein lactose permease (LP), may be incorporated into liposome bilayers thus allowing coupling of sugar proton transport with a stoichiometric ratio of $1: 1$, as demonstrated with the fluorescent $\mathrm{pH}$-probe pyranine entrapped in these liposomes Kiefer et al., 1991. These LP-containing liposomes have been incorporated within planar lipid bilayer coatings of an ISFET gate sensitive to $\mathrm{pH}$. Preliminary results have shown that these modified ISFETs enable rapid and reversible detection of lactose in an FIA system. Protein receptor based biosensors have been recently developed Sugawara et al., 1997b. The result of the binding of the analyte, here named agonist, to immobilized channel receptor proteins, is monitored by changes in ion fluxes through these channels. For example glutamate, as target agonist, may be determined in the presence of various interfering agonists, by detecting $\mathrm{Na}^{+}$or $\mathrm{Ca}^{2+}$ fluxes, using conductivity or ion selective electrodes. Due to the dependence of ion channel switching on agonist binding, there is usually no need for enzyme labelling of the receptor to achieve the desired sensitivity.

A developing field in electrochemical biosensors is the use of chips and electrochemical methods to detect binding of oligonucleotides (gene probes) (Table 1). There are two approaches currently developed. The fist one intercalates into the oligonucleotide duplex, during the formation of a double stranded DNA on the probe surface, a molecule that is electroactive. The second approach directly detects guanine that is electroactive.

In conclusion, biocomplex-based biosensors although showing promising behaviour, have not yet reached the advanced development stage of the biocatalyst-based systems. Being based on equilibrium reactions, they generally present a very narrow linear operating range of concentration and are often unable to monitor continuously the analyte concentration. Furthermore, some of these biosensors may be difficult to operate in a 
biological matrix because their sensing layer has to be in direct contact with the sample and because it may not be possible to incorporate an outer membrane to separate the sensing element from the sample matrix.

\subsection{Detection or measurement mode: electrochemical transduction or detection}

\subsubsection{Amperometry}

Amperometry is based on the measurement of the current resulting from the electrochemical oxidation or reduction of an electroactive species. It is usually performed by maintaining a constant potential at a $\mathrm{Pt}, \mathrm{Au}$ or $\mathrm{C}$ based working electrode or on array of electrodes with respect to a reference electrode, which may also serve as the auxiliary electrode, if currents are low (from $10^{-9}$ to $10^{-6} \mathrm{~A}$ ). The resulting current is directly correlated to the bulk concentration of the electroactive species or its production or consumption rate within the adjacent biocatalytic layer. As biocatalytic reaction rates are often chosen to be first order dependent on the bulk analyte concentration, such steady-state currents are usually proportional to the bulk analyte concentration.

\subsubsection{Potentiometry}

Potentiometric measurements involve determination of the potential difference between either an indicator and a reference electrode, or two reference electrodes separated by a permselective membrane, when there is no significant current flowing between them. The transducer may be an ion-selective electrode (ISE) which is an electrochemical sensor based on thin films or selective membranes as recognition elements Buck and Lindner, 1994. The most common potentiometric devices are $\mathrm{pH}$ electrodes; several other ion $\left(\mathrm{F}^{-}, \mathrm{I}^{-}\right.$, $\left.\mathrm{CN}^{-}, \mathrm{Na}^{+}, \mathrm{K}^{+}, \mathrm{Ca}^{2+}, \mathrm{NH}_{4}^{+}\right)$or gas $\left(\mathrm{CO}_{2}, \mathrm{NH}_{3}\right)$ selective electrodes are available. The potential differences between these indicator and reference electrodes are proportional to the logarithm of the ion activity or gas fugacity (or concentration), as described by the Nernst-Donnan equation. This is only the case when (i) the membrane or layer selectivity is infinite or if there is a constant or low enough concentration of interfering ions; and (ii) potential differences at various phase boundaries are either negligible or constant, except at the membrane/sample-solution boundary.

When a biocatalyst layer is placed adjacent to the potentiometric detector, one has to take into account of, as for any biocatalyst sensor: (1) transport of the substrate to be analysed to the biosensor surface; (2) analyte diffusion to the reacting layer; (3) analyte reaction in the presence of biocatalyst and (4) diffusion of reaction product towards both the detector and the bulk solution. The response of potentiometric biocatalytic sensors is, as for amperometric biosensors, either steady-state or transient, but it is never an equilibrium response. The situation is more complex for enzyme-labelled immuno-sensors: although the Ab-Ag complex is expected to reach an equilibrium and reactions to be either reversible or irreversible, the labelled enzyme activity is measured under steady-state analyte consumption conditions.

Another important feature of the ISE based biosensors, such as $\mathrm{pH}$ electrodes, is the large dependence of their response on the buffer capacity of the sample (see Section 4.2) and on its ionic strength.

\subsubsection{Surface charge using field-effect transistors (FETS)}

An important variation of the systems used to determine ion concentrations are the ion-sensitive field-effect transistors (ISFETs). An ISFET is composed of an ion-selective membrane applied directly to the insulated gate of the FET Covington, 1994. When such ISFETs are coupled with a biocatalytic or biocomplexing layer, they become biosensors, and are usually called either enzyme (ENFETs) or immunological (IMFETs) fieldeffect transistors. Operating properties of ENFET and IMFET-based devices are strongly related to those of the ISE based biosensors.

\subsubsection{Conductometry}

Many enzyme reactions, such as that of urease, and many biological membrane receptors may be monitored by ion conductometric or impedimetric devices, using interdigitated microelectrodes Cullen et al., 1990. Because the sensitivity of the measurement is hindered by the parallel conductance of the sample solution, usually a differential measurement is performed between a sensor with enzyme and an identical one without enzyme.

\section{Analytes or Reactions monitored}

Biosensors may be further classified according to the analytes or reactions that they monitor. One should clearly differentiate between the direct monitoring of analytes, or of biological activity, and the indirect monitoring of inhibitors.

\subsection{Direct monitoring of analyte, or, alternatively, of biological activity producing or consuming analytes}

Direct monitoring of analytes has clearly been the major application of biosensors. Nevertheless, one should be aware that the same biosensor can be a useful tool also for the direct monitoring of enzyme or living cell activities, by measuring, continuously or sequentially, the production or consumption of a given compound. 


\subsection{Indirect monitoring of inhibitor or activator of the biochemical receptor}

Alternatively, biosensors have been developed for indirect monitoring of organic pesticides, or inorganic (heavy metals, fluoride, cyanide, etc.) substances which inhibit biocatalytic properties of the biosensor. However such devices are often irreversible. As for immunosensors, their original biological activity can be usually restored only after chemical treatment and such sensors are not classified as reagentless devices. Their potential use, especially for environmental monitoring, is thus often more as a warning system, not requiring exact measurement of the analyte concentration. We recommend that they be referred to as single-use biosensors, except when they can be rapidly and reproducibly regenerated, such as the cyanide biosensor using the inhibition of a cytochrome oxidase which is regenerated by washing with phosphate buffer at $\mathrm{pH} 6.3$ Amine et al., 1995.

\section{Biosensor construction}

\subsection{Immobilization of biological receptors}

Since the development of the enzyme-based sensor for glucose, first described by Clark in 1962, in which glucose oxidase was entrapped between two membranes Clark et al., 1962, an impressive literature on methods of immobilization and related biosensor development has appeared. These methods have been extensively reviewed elsewhere Turner et al., 1987; Guilbault, 1984; Mosbach and (Ed.), 1988; Cass and (Ed.), 1990; Göpel et al., 1991; Blum et al., 1991; Kas et al., 1996. Biological receptors, i.e. enzymes, antibodies, cells or tissues, with high biological activity, can be immobilized in a thin layer at the transducer surface by using different procedures. The following procedures are the most generally employed:

1. Entrapment behind a membrane: a solution of enzyme, a suspension of cells or a slice of tissue is, simply, confined by an analyte permeable membrane as a thin film covering the electrochemical detector;

2. Entrapment of biological receptors within a polymeric matrix, such as polyacrylonitrile, agar gel, polyurethane (PU) or poly(vinyl-alcohol) (PVAL) membranes, sol gels or redox hydrogels with redox centers such as $\left[\mathrm{Os}(\mathrm{bpy})_{2} \mathrm{Cl}\right]^{+/ 2+}$ Rajagopalan et al., 1996;

3. Entrapment of biological receptors within self assembled monolayers (SAMs) or bilayer lipid membranes (BLMs);

4. Covalent bonding of receptors on membranes or surfaces activated by means of bifunctional groups or spacers, such as glutaraldehyde, carbodiimide,
SAMs or multilayers, avidin-biotin silanization, some of such activated membranes being commercially available;

5. Bulk modification of entire electrode material, e.g. enzyme modified carbon paste or graphite epoxy resin Gorton, 1995.

Receptors are immobilized either alone or they are mixed with other proteins, such as bovine serum albumin (BSA), either directly on the transducer surface, or on a polymer membrane covering it. In the latter case, preactivated membranes can be used directly for the enzyme or antibody immobilization without further chemical modification of the membrane or macromolecule.

Apart from the last example, reticulation and covalent attachment procedures are more complicated than entrapment ones, but are especially useful in cases where the sensor is so small that the appropriate membrane must be fabricated directly on the transducer. Under such conditions more stable and reproducible activities can be obtained with covalent attachment.

\subsection{Inner and outer membranes}

Besides the reacting layer or membrane, many biosensors, especially those designed for biological or clinical applications, incorporate one or several inner or outer layers. These membranes serve three important functions:

1. Protective barrier. The outer membrane prevents large molecules, such as proteins or cells of biological samples, from entering and interfering with the reaction layer. It also reduces leakage of the reacting layer components into the sample solution. This function of the outer membrane is important, for example, for implanted glucose sensors, since its glucose oxidase is of non-human origin and may cause immunological reactions. Furthermore, a properly chosen membrane exhibits permselective properties, which may be additionally beneficial to the biosensor function. It may decrease the influence of possible interfering species detected by the transducer. For example, most in vivo or ex vivo glucose biosensors present a negatively charged inner cellulose acetate membrane in order to decrease the interfering effect of ascorbate or urate, electrochemically detected together with enzymatically generated hydrogen peroxide.

2. Diffusional outer barrier for the substrate. As most enzymes follow some form of Michaelis-Menten kinetics, enzymatic reaction rates are largely nonlinear with concentration. Nevertheless, linear dynamic ranges may be large if the sensor response is controlled by the substrate diffusion through the membrane and not by the enzyme kinetics. This control is achieved by placing a thin outer mem- 
brane over a highly active enzyme layer Scheller and Pfeiffer, 1978; Bindra et al., 1991: the thinner is this membrane, the shorter is the biosensor response time. Furthermore, such diffusional barrier also makes the sensor response independent of the amount of active enzyme present and improves the sensor response stability.

3. Biocompatible and biostable surfaces. Biosensors are subject to two sets of modifications when they are in direct contact with biological tissues or fluids, i.e. implanted in vivo or, more generally, in biologically active matrices, such as cell cultures:

$\circ$ modification of the host biological sample by various reactions caused by biosensor introduction and toxicity, mutagenicity, carcinogenicity, thrombogenicity or immunogenicity of its elements,

modification of the biosensor operating properties by sample components or structure: external layer or inner detector fouling, inhibition of the biorecognition reaction, substrate and/or co-substrate transport rate towards the biorecognition area.

Apart from molecular recognition systems or transducers which require direct contact between sample and biological receptor, the choice of an outer layer is generally essential for the stability of the response after implantation. Depending upon sensor diameter, i.e. centimeter or sub-millimeter range, pre-cast membranes, such as those made of collagen, polycarbonate or cellulose acetate, or, alternatively, polymeric materials deposited by dip- or spin-coating (cellulose acetate, Nafion or polyurethane) may be used. Microsize biosensors are often prepared by entrapping the enzyme by an electropolymerization step.

If the implantation of the biosensor does not materially affect the normal functioning of the host medium and if the medium does not materially affect the normal operation of the biosensor, then the biosensor is considered to be biocompatible.

\section{Performance criteria: guidelines for reporting characteristics of the biosensor response}

As for any sensor based on molecular recognition Buck and Lindner, 1994, it is important to characterize a biosensor response: it is even more important here since operating parameters may indicate the nature of the rate-limiting steps (transport or reaction) and facilitate biosensor optimization in a given matrix. This section will briefly list main performance criteria and discuss their relation to properties of the receptor and transducer parts of electrochemical biosensors. When performance criteria are not specific to biosensors but common to most types of chemical sensors or analytical methods, e.g. precision, accuracy, interlaboratory and interpersonal reproducibility, it is recommended that standard IUPAC definitions be followed Inczedy et al., 1998; Buck and Lindner, 1994.

Most of the discussion below relates to enzyme-based biosensors. In the case of immunosensors, a key issue is the capture capacity of the surface, i.e. the number of molecules on the surface which are actually biologically active. One of the methods for assessing this parameter consists in measuring the specific activity, i.e. the ratio of the number of active molecules/the total number of immobilized molecules. This figure is very dependent on the mode of immobilization (molecular orientation, number of points of attachment) and can range from about 0.15 to 0.3 , rarely reaching 1 . This capture capacity becomes especially important when the surface is decreased, as in microfluidic applications. Another important issue for immunosensors is the question of whether the surface can be regenerated without significant loss of activity (see Section 2.1.2).

The rapid proliferation of biosensors and their diversity has led to a lack of rigour in defining performance criteria. Although each sensor can only truly be evaluated for a particular application, it is still useful to establish standard protocols for evaluation of performance criteria, in accordance with standard IUPAC protocols or definitions Inczedy et al., 1998. These protocols are recommended for general use and include four sets of parameters, described below.

\subsection{Calibration characteristics: sensitivity, working and linear concentration range, detection and quantitative determination limits}

Sensor calibration is performed, in general, by adding standard solutions of the analyte and by plotting steady-state responses $R_{\mathrm{ss}}$, possibly corrected for a blank (often called background) signal $R_{\mathrm{bl}}$, versus the analyte concentration, $c$, or its logarithm, $\log c / c^{\circ}$, where $c^{\circ}$ refers to a reference concentration, usually 1 mol $1^{-1}$, although such high concentration value is never used, the highest values reaching usually $1-10$ mmol $1^{-1}$. Transient responses are important for sequential samples but are less significant for continuous monitoring: within several possibilities, they are generally defined as the maximum rates of variation of the sensor response $(\mathrm{d} R / \mathrm{d} t)_{\max }$, after addition of analyte into the measurement cell. A convenient way to perform such calibrations, under well-defined hydrodynamic conditions, is to place the biosensor in a FIA system for sequential sample analysis.

The sensitivity and linear concentration range of steady-state calibration curves are determined by plotting the ratio $\left(R_{\mathrm{ss}}-R_{\mathrm{bl}}\right) / c$ or $\left(R_{\mathrm{ss}}-\mathrm{R}_{\mathrm{bl}}\right) / \log c / c^{\circ}$ versus $\log c / c^{\circ}$. This method is much more concise than plotting the usual calibration curves $\left(R_{\mathrm{ss}}-R_{\mathrm{bl}}\right)$ versus $c$ 
or $\log c / c^{\circ}$ since it gives the same weight to low and high analyte concentration results. Likewise, sensitivity and linear range of transient calibration curves are determined by plotting the ratio $(\mathrm{d} R / \mathrm{d} t)_{\max } / c$ or $(\mathrm{d} R /$ $\mathrm{d} t)_{\max } / \log c / c^{\circ}$ versus $\log c / c^{\circ}$. In both cases sensitivity is to be determined within the linear concentration range of the biosensor calibration curve.

Electrochemical biosensors always have an upper limit of the linear concentration range. This limit is directly related to the biocatalytic or biocomplexing properties of the biochemical or biological receptor, although in the case of enzyme-based biosensors, it may be significantly extended by using an outer layer diffusion barrier to substrate $\mathrm{S}$ (see Section 4.2.). The compromise for such an extension in the linear concentration range is, obviously, the decrease of sensor sensitivity. The local substrate concentration, within the reaction layer, can be at least two orders of magnitude lower than in the bulk solution. In relation to the usual parameters for Michaelis-Menten kinetics, i.e. $K_{\mathrm{M}}$ and $V_{\max }$, enzyme based biosensors are often characterized by their apparent $K_{\mathrm{M}}$ and $\left(R_{\mathrm{ss}}-R_{\mathrm{bl}}\right)_{\max }$ : the first parameter represents the analyte concentration yielding a response equal to half of its maximum value, $\left(R_{\mathrm{ss}}-\right.$ $\left.R_{\mathrm{bl}}\right)_{\max }$ for infinite analyte concentration. When the apparent $K_{\mathrm{M}}$ is much larger than its value for soluble enzyme, it means either that a significant substrate diffusion barrier is present between the sample and the reaction layer, or that the rate of reaction of the co-substrate, $\mathrm{S}^{\prime}$, with the enzyme is increased. As for enzyme solution kinetics, the apparent $K_{\mathrm{M}}$ is usually determined by Lineweaver-Burk reciprocal plots, i.e. $1 /\left(R_{\mathrm{ss}}-R_{\mathrm{b} 1}\right)$ versus $1 / c$. As for any electrochemical sensor, one should state the composition and the number of standards used and how the sample matrix is simulated or duplicated. It may be necessary to specify procedures for each biosensor type and application. This is especially important for single-use biosensors based on immuno affinity (see Section 2.1.2) or on inhibition reactions (see Section 3.2).

The sensitivity is the slope of the calibration curve, i.e. $\left(R_{\mathrm{ss}}-R_{\mathrm{bl}}\right)$ versus $c$ or $\log c / c^{\circ}$. One should always avoid confusion between sensitivity and detection limits. The limit of detection (LOD) and of quantification (LOQ) take into account the blank and the signal fluctuation (noise). Their definition is not specific to biosensors and IUPAC recommendations should be used. The working concentration range, which may considerably extend the linear concentration range, is determined by the lower and upper limits of quantification.

\subsection{Selectivity and reliability}

Biosensor selectivity is determined and expressed as for other amperometric or potentiometric sensors Mc-
Naught and Wilkinson, 1997; Umezawa et al., 1995. It depends both upon the choice of biological receptor and transducer. Many enzymes are specific. Nevertheless, class (non-selective) enzymes, such as alcohol, group sugar or amino-acid oxidases, peroxidases, laccase, tyrosinase, ceruloplasmin, alcohol or glucose NAD-dehydrogenase, etc, have been used for the development of class biosensors, such as those for determination of phenols, used in environmental monitoring or food analysis. Bacteria, yeast or tissue cultures are naturally non-specific. Whereas oxygen electrodes, $\mathrm{pH}$ electrodes and ISFETs show appropriate selectivity, metal electrodes are often sensitive to numerous interfering substances. This direct selectivity can be modified when these transducers are associated with receptors. For example, when $\mathrm{pH}$-sensitive ENFETs are used as transducers, their responses are influenced by the buffer capacity of the sample, since part of the released protons react with the buffer components and only the remainder is sensed by the transducer. In this case, it is, in fact, the sensitivity of the biosensor, which is modified, and not its selectivity.

When transducer interfering substances are well identified, such as ascorbate or urate in glucose sensors based on hydrogen peroxide detection, their influence may be restricted by the application of appropriate inner or outer membranes (see Section 4.2.). Alternatively, a compensating sensor may be introduced in the set-up, without biological receptor on its surface Thévenot et al., 1979. Such a differential design is frequently used for ISFET- or ENFET-based sensors. Within various methods for biosensor selectivity determination, two are recommended depending upon the aim of its measurement. The first one consists in measuring the biosensor response to interfering substance addition: a calibration curve for each interfering substance is plotted and compared to the analyte calibration curve, under identical operating conditions. Selectivity is expressed as the ratio of the signal output with the analyte alone and with the interfering substance alone, at the same concentration as that of the analyte. In the second procedure interfering substances are added, at their expected concentration, into the measuring cell, already containing usual analyte concentration, at the mid-range of its expected value. Selectivity is then expressed as the percentage of variation of the biosensor response: although more easily quantified than the calibration curve comparison performed in the first procedure, the second method is characteristic of each application and presents a more restricted significance. Such selectivity may depend on the analyte concentration range that is determined.

The reliability of biosensors for given samples depends both on their selectivity and their reproducibility. It has to be determined under actual operating conditions, i.e. in the presence of possible interfering sub- 
stances. In order to be reliable for an analyst, the biosensor response should be directly related to the analyte concentration and should not vary with fluctuations of concentrations of interfering substances within the sample matrix. Thus, for each type of biosensor and sample matrix, one should clearly state the reasonable interference that should be considered and how its influence should be quantified. This reliability determination is necessary for accuracy assessment for each application.

\subsection{Steady-state and transient response times, sample throughput}

Steady-state response time is easily determined for each analyte addition into the measurement cell. It is the time necessary to reach $90 \%$ of the steady-state response Lindner et al., 1986. Transient response time corresponds to the time necessary for the first derivative of the output signal to reach its maximum value $(\mathrm{d} R /$ $\mathrm{d} t)_{\max }$ following the analyte addition. Both response times depend upon the analyte, co-substrate and product transport rates through different layers or membranes. Therefore, the thickness and permeability of these layers are essential parameters. Both response times also depend upon the activity of the molecular recognition system. The higher this activity, the shorter is this response time. Finally, they also depend upon the mixing conditions of the sample into the batch measurement cell: such mixing time may not be negligible. A simple way to better define such hydrodynamic conditions in the biosensors vicinity is to use a FIA system for sample introduction. When biosensors are part of FIA systems, their response time is defined as for any other FIA detector: if the analyte concentration is varied stepwise, steady-state and transient response times are defined as in batch; alternatively, if analyte pulses are introduced into the circulating fluid, only transient responses are available. Finally, when sensors are implanted in vivo or placed in or in the vicinity of industrial reactors, their operational response time also incorporates the analyte and co-substrate transport rates towards the sensor site.

When biosensors are used for sequential measurements, either in batch or flow-through set-ups, the sample throughput is a measure of the number of individual samples per unit of time. This parameter takes into account the steady-state or transient response times but also includes the recovery time, i.e. the time needed for the signal to return to its base line.

Both types of response times, as well as sample throughput, may depend on sample composition, analyte concentration, or the sensor history: such dependencies should be tested and quantified.

Theoretical modelling of biosensor operation enables a better understanding of the relative importance of the factors mentioned above on response time Eddowes, 1990. Modeling is somewhat limited by the necessary knowledge of a large number of sensor parameters (thickness, partition and diffusion coefficients of each membrane or layer for each species, distribution of biocatalytic or biocomplexing activity within the sensor layers, transducer operating properties, etc.). Often, such modelling is restricted to steady-state operation and is not sufficiently advanced for the evaluation of transient responses and response in general Albery et al., 1987.

\subsection{Reproducibility, stability and lifetime}

Definition of reproducibility is the same for electrochemical biosensors as for any other analytical device: reproducibility is a measure of the scatter or the drift in a series of observations or results performed over a period of time. It is generally determined for the analyte concentrations within the usable range.

The operational stability of a biosensor response may vary considerably depending on the sensor geometry, method of preparation, as well as on the applied receptor and transducer. Furthermore it is strongly dependent upon the response rate limiting factor, i.e. a substrate external or inner diffusion or biological recognition reaction. Finally, it may vary considerably depending on the operational conditions. For operational stability determination, we recommend consideration of the analyte concentration, the continuous or sequential contact of the biosensor with the analyte solution, temperature, $\mathrm{pH}$, buffer composition, presence of organic solvents, and sample matrix composition. Although some biosensors have been reported usable under laboratory conditions for more than one year, their practical lifetime is either unknown or limited to days or weeks when they are incorporated into industrial processes or to biological tissue, such as glucose biosensors implanted in vivo Pickup and Thévenot, 1993. For storage stability assessment, significant parameters are the state of storage, i.e. dry or wet, the atmosphere composition, i.e. air or nitrogen, $\mathrm{pH}$, buffer composition and presence of additives.

While it is relatively easy to determine the laboratory bench stability of biosensors, both during storage and operation in the presence of analyte, procedures for assessing their behaviour during several days of introduction into industrial reactors is much more complex and difficult to handle. In both cases, i.e. bench or industrial set-ups, it is necessary to specify whether lifetime is a storage (shelf) or operational (use) lifetime and what the storage and operating conditions were, and specify substrate(s) concentration(s), as compared to the apparent Michaelis-Menten constant $K_{\mathrm{M}}$ (see Section 5.1). Knowledge of the biosensor rate limiting step or factor is especially important for the understanding of stability properties. 
Finally, the mode of assessment of lifetime should be specified, i.e. by reference to initial sensitivity, upper limit of the linear concentration range for the calibration curve, accuracy or reproducibility. We recommend the definition of lifetime, noted $t_{\mathrm{L}}$, as the storage or operational time necessary for the sensitivity, within the linear concentration range, to decrease by a factor of 10 $\left(t_{\mathrm{L} 10}\right)$ or $50 \%\left(t_{\mathrm{L} 50}\right)$. For the determination of the storage lifetime, we suggest comparison of sensitivities of different biosensors, derived from the same production batch, after different storage time under identical conditions. Biosensor stability may also be quantified as the drift, when the sensitivity evolution is monitored during either storage or operational conditions. The drift determination is especially useful for biosensors which evolution is either very slow or studied during rather short period of time.

\section{Conclusion}

Some characteristics of biosensors are common to different types of electrochemical sensors. Others are more specific to biosensor principles but may be common to different types of transducers. Responses of biosensors will be controlled by kinetics of recognition and transduction reactions, or by mass transfer rates. Determination of the rate-limiting step is clearly essential for the understanding, optimization and control of such biosensor performance criteria.

As with most nomenclature documents on complex technological developments, the definitions, terminology, and classification of electrochemical biosensors cannot unambiguously address every detail, nuance and contingency of this diverse subject. There will invariably be exceptions to some of the nomenclature and classification recommendations. However, this is a living document and, as such, will be revised periodically as needed to address ambiguities and new technological developments as they arise in the evolution of electrochemical biosensors. Comments on this document are actively solicited from scientists working in this, and related, fields of research.

\section{References}

Aizawa, M., 1991. Anal. Chim. Acta 250, 249.

Albery, W.J., Craston, D.H., 1987. In: Turner, A.P.F., Karube, I., Wilson, O.S. (Eds.), Biosensors: fundamentals and applications. Oxford University Press, Oxford, p. 180.
Amine, A., Alafandy, M., Kauffmann, J.M., 1995. Anal. Chem. 67, 2822.

Bartlett, P.N., Tebbutt, P., Whitaker, R.G., 1991. Prog. React. Kinet. $16,55$.

Bergveld, P., Thévenot, D.R., 1993. In: Turner, A.P.F. (Ed.), Advances in biosensors, Supplement 1. JAI Press, p. 31.

Bindra, D.S., Zhang, Y., Wilson, G.S., Sternberg, R., Thévenot, D.R., Moatti, D., Reach, G., 1991. Anal. Chem. 63, 1692.

Blum, L.J., Coulet, P.R. (Eds.), 1991. Biosensor principles and applications. Marcel Dekker, New York.

Buck, R.P., Lindner, E., 1994. Pure Appl. Chem. 66, 2527.

Cammann, K., 1977. Fresenius Z. Anal. Chem. 287, 1.

Cass, A.E.G. (Ed.), 1990. Biosensors: a practical approach. I.R.L. Press, Oxford.

Clark, L.C., Lyons, J.R., Lyons, C., 1962. Ann. N.Y. Acad. Sci. 102, 29.

Covington, A.K., 1994. Pure Appl. Chem. 66 (3), 565.

Cullen, D.C., Sethi, R.S., Lowe, C.R., 1990. Anal. Chim. Acta 231, 33.

Durst, R.A., Bäumner, A.J., Murray, R.W., Buck, R.P., Andrieux, C.P., 1997. Pure Appl. Chem. 69, 1317.

Eddowes, M.J., 1990. In: Cass, A.E.G. (Ed.), Biosensors: a practical approach. I.R.L. Press, Oxford, pp. 211.

Göpel, W., Jones, T.A., Kleitz, M., Lundström, I., Seiyama, T., 1991. Chemical and biochemical sensors. In: Göpel, W., Hesse, H., Zemel, J.N. (Eds.), Sensors - a comprehensive survey, vols. 2-3. $\mathrm{VCH}$, New York.

Gorton, L., 1995. Electroanal. 7, 23.

Guilbault, G.G., 1984. Handbook of immobilized enzymes. Marcel Dekker, New York.

Inczedy, J., Lengyel, T., Ure, A.M. (Eds.), 1998. JUPAC compendium of analytical nomenclature. 3rd edition, Blackwell Science, Oxford.

Kas, J., Marek, M., Stastny, M., Voll, R., 1996. Chapter 6. In: Brabec, V., Valz, D., Milazzo, G. (Eds.), Experimental techniques in bioelectrochemistiy. Birkäuser Verlag, Basel, pp. 361-447.

Kiefer, H., Klee, B., John, E., Stierhof, Y.D., Jähnig, F., 1991. Biosens. Bioelectron. 6, 233.

Kutner, W., Wang, J., L’Her, M., Buck, R.P., 1998. Pure Appl. Chem. 70 (6), 1301.

Lindner, E., Toth, K., Pungor, E., 1986. Pure Appl. Chem. 58 (3), 469.

McNaught, A.D., Wilkinson, A., 1997. Compendium of chemical terminology, Second edition. Blackwell Science, Oxford.

Mosbach, K. (Ed.), 1988. Methods in enzymology. Vol.137, Academic Press, New York.

Pickup, J., Thévenot, D.R., 1993. In: Turner, A.P.F. (Ed.), Chemical sensors for in vivo monitoring: advances in biosensors. Supplement 1, JAI Press, pp. 201.

Rajagopalan, R., Aoki, A., Heller, A., 1996. J. Phys. Chem. 100, 3719.

Scheller, F.W., Pfeiffer, D., 1978. Z. Chem. 18, 50.

Sugawara, M., Sato, H., Ozawa, T., Umezawa, Y., 1997a. In: Scheller, F.W., Scubert, F., Fedrowitz, J. (Eds.), Frontiers in biosensorics - fundamental aspects. Birkhäuser Verlag, Basel, pp. 121-131.

Sugawara, M., Hirano, A., Rehak, M., Nakanishi, J., Kawai, K., Sato, H., Umezawa, Y., 1997b. Biosens. Bioelectron. 12 (5), 425.

Thévenot, D.R., Coulet, P.R., Sternberg, R., Laurent, J., Gautheron, D.C., 1979. Anal. Chem. 51, 96.

Turner, A.P.F., Karube, I., Wilson, G.S. (Eds.), 1987. Biosensors, fundamentals and applications. Oxford University Press, Oxford.

Umezawa, Y., Umezawa, K., Sato, H., 1995. Pure Appl. Chem. 67, 507.

Wollenberger, U., Schubert, F., Scheller, F.W., 1993. Trends Biotechnol. $11,255$. 